\title{
Statin adherence and persistence on secondary prevention of cardiovascular disease in Taiwan
}

\author{
Wen-Yi Shau, ${ }^{\oplus 1}$ Chao-Lun Lai, ${ }^{2,3}$ Shih-Ting Huang, ${ }^{2}$ Shu-Ting Chen, ${ }^{2} \operatorname{Jim} \mathrm{Z} \mathrm{Li,}{ }^{4}$ \\ Selwyn Fung, ${ }^{5}$ Vicki C Tse, ${ }^{6}$ Mei-Shu Lai ${ }^{2}$
}

- Additional material is published online only. To view please visit the journal online (http://dx.doi.org/10.1136/ heartasia-2018-011176).

${ }^{1}$ Medical Affairs, Pfizer Taiwan, New Taipei, Taiwan

${ }^{2}$ Institute of Epidemiology and Preventive Medicine, College of Public Health, National Taiwan University, Taipei, Taiwan ${ }^{3}$ Department of Internal Medicine and Center for Critical Care Medicine, National Taiwan University Hospital Hsin-Chu Branch, Hsin-Chu, Taiwan ${ }^{4}$ Global Health and Value, Pfizer, San Diego, California, USA ${ }^{5} A P A C$ regional Medical Affairs, Pfizer, New York City, New York, USA

${ }^{6}$ APCER Life Sciences, Princeton, New Jersey, USA

\section{Correspondence to}

Professor Mei-Shu Lai, Institute of Epidemiology and Preventive Medicine, College of Public Health, National Taiwan University, Taipei 10617, Taiwan;

Received 4 January 2019 Revised 2 May 2019 Accepted 4 May 2019 mslai@ntu.edu.tw

\section{ABSTRACT}

Background Evidence and treatment guidelines support the use of statins in patients with established atherosclerotic cardiovascular disease (ASCVD) for secondary prevention of subsequent cardiovascular (CV) event. However, treatment adherence and persistence are still a concern.

Methods We constructed a retrospective populationbased cohort of patients, who initiated statin treatment within 90 days after discharge from hospital for ASCVD using the claims database of Taiwan National Health Insurance. Proportion of days covered (PDC) was used to measure statin adherence, and PDC $\geq 80 \%$ was defined as good adherence. The study outcomes were subsequent rehospitalisation or in-hospital death due to composite ASCVD, myocardial infarction or ischaemic stroke. Their associations with statin prescription adherence or persistence were analysed using time-dependent Cox proportional hazards model.

Results The study cohort included 185252 postdischarge statin initiators. There were 50015 subsequent ASCVD rehospitalisations including 2858 in-hospital death during 7 years of study period. Good adherence was significantly associated with lower risk of ASCVD rehospitalisation (adjusted HR (aHR) 0.90; $95 \% \mathrm{Cl} 0.87$ to 0.92 ) and significantly lower risk of in-hospital death (aHR 0.59; $95 \% \mathrm{Cl} 0.53$ to 0.65). Compared with constant use of statin, patients in the three less persistent states (recent stop, non-persistence and intermittent use) were associated with higher risk of subsequent ASCVD rehospitalisation, aHRs were 1.16, 1.13 and 1.26 , respectively (all $p<0.05$ ). The increased risks were consistent with specific outcome of acute myocardial infarction and ischaemic stroke. Also, patients in the recent stop period had significantly higher risk for fatal CV event.

Conclusions Good adherence and persistence to statin therapy are significantly associated with lower risk of secondary ASCVD rehospitalisation and in-hospital death.

\section{INTRODUCTION}

Atherosclerotic cardiovascular disease (ASCVD) Check for updates

(C) Author(s) (or their employer(s)) 2019. No commercial re-use. See rights and permissions. Published by BMJ.

To cite: Shau W-Y, Lai C-L, Huang S-T, et al. Heart

Asia 2019;11:e011176.

doi:10.1136/

heartasia-2018-011176 comprises several disorders that are of atherosclerotic origin, ${ }^{1}$ which leads to significant health and economic burdens. ${ }^{2}$ Among modifiable risk factors, hyperlipidaemia is one of the foremost risk factors for ASCVD. ${ }^{3} 4$ Landmark randomised controlled clinical trials (RCT) have established that patients on high-intensity statins have a lower incidence of secondary ASCVD. ${ }^{5-7}$ A systematic review of RCTs further confirmed the efficacy of statin therapy in preventing ASCVD. ${ }^{8}$ In recent years, several international lipid treatment guidelines ${ }^{9} 10$ have

\section{Key messages}

What is already known about this subject?

- Despite the solid clinical evidence and strong recommendations for the long-term use of statins in secondary prevention of ASCVD, adherence and persistence are still issues that impact the treatment effect in real-world clinical practice.

\section{What does this study add?}

- As most of the studies for statin adherence were done in western countries, this study add information on Asian population.

\section{How might this impact on clinical practice?}

- Good adherence and persistence to statin therapy are significantly associated with lower risk of secondary ASCVD rehospitalisation and in-hospital death; optimising patient adherence and persistence behavior will improve treatment outcomes.

consistently recommended intensive statin therapy for patients with ASCVD to prevent subsequent cardiovascular (CV) events.

Despite the solid clinical evidence and strong recommendations for the long-term use of statins in secondary prevention of ASCVD, adherence and persistence are still issues that impact the treatment effect in real-world clinical practice. Poor adherence or non-persistence to statins increases the risk for CV events and mortality in patients with ASCVD history. ${ }^{11} 12$ A systematic review of 28 real-world studies published in 2014 showed that non-adherence and non-persistence to statin treatment significantly increased the relative risk of secondary $\mathrm{CV}$ events and mortality ranging from 1.22 to $5.26 .^{13}$ However, most of the studies reviewed were conducted in western countries. The information of statin treatment adherence and persistence in patients with ASCVD for secondary prevention of $\mathrm{CV}$ disease is limited in Asian population. Thus, the aim of this study was to assess the effect of statin treatment adherence and persistence on the prevention of subsequent $\mathrm{CV}$ events in patients discharged from hospital for a new-onset ASCVD in a realworld setting in Taiwan.

\section{METHODS}

\section{Study design}

We constructed a retrospective population-based cohort using the claims database of National Health 


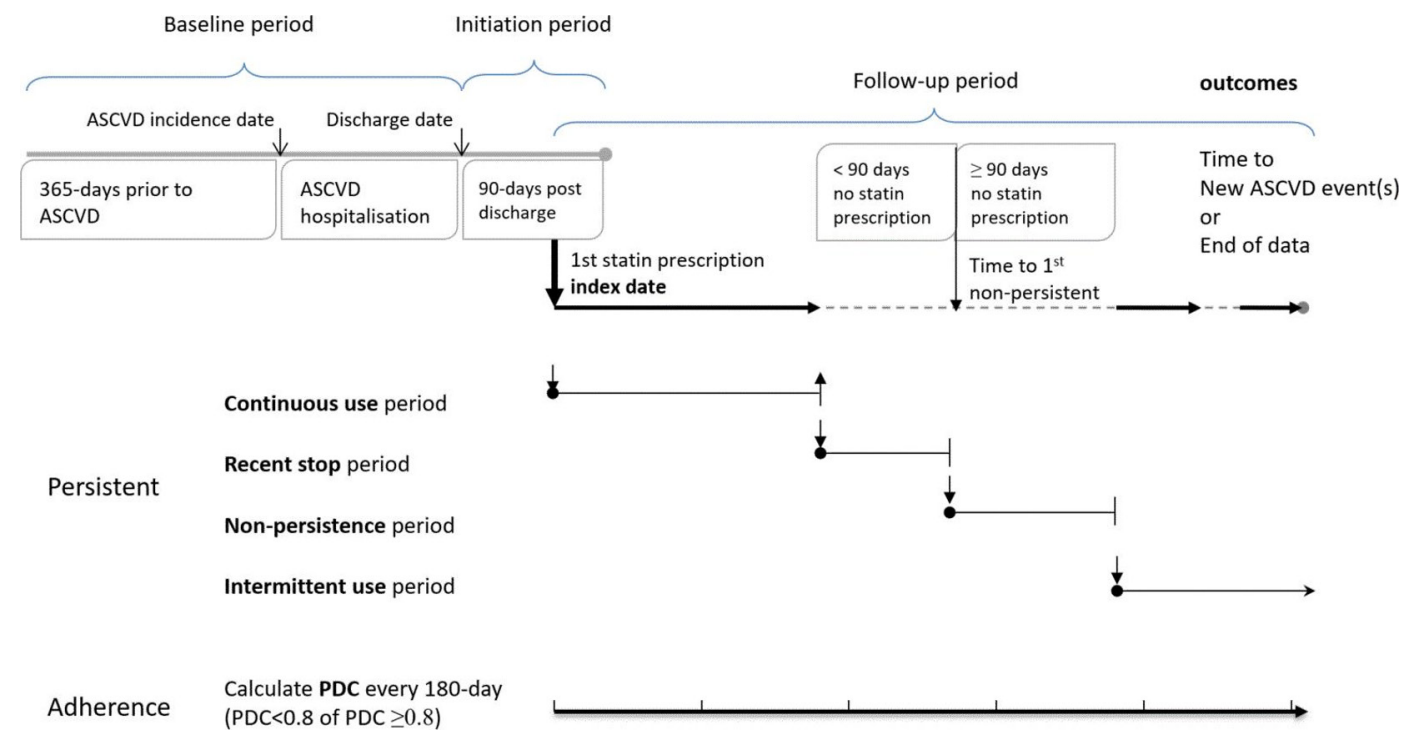

Figure 1 Study design schematic. ASCVD, atherosclerotic cardiovascular disease; PDC, proportion of days covered.

Insurance (NHI) in Taiwan. ${ }^{14}$ Figure 1 shows the study design schematic and definitions of statin adherence and persistence measurements.

\section{Data source and study cohort}

The Taiwan NHI programme was implemented on 1 March 1995. This is a compulsory universal programme. In 2014, over $99 \%$ of all the 23 million population in Taiwan were enrolled. The programme provides coverage including ambulatory care, emergency visits, hospitalisation, surgical procedures, laboratory tests, diagnostic examinations, dental services, traditional Chinese medicines and prescription medicines. The reimbursement claims data are captured through an electronic system and updated on a daily basis. This database provides a great resource for population-based research and has been widely used for various clinical and epidemiological studies.

The date of the first statin prescription within 90 days of patients discharged from hospital for new-onset ASCVD was defined as the index date. We identified cases with index dates between 1 January 2006 and 31 December 2012 from the NHI claims database. All data in the 1-year period prior to the ASCVD hospitalisation were examined to confirm it was a new-onset episode. ASCVD events were identified by hospital discharge diagnosis and in-hospital revascularisation procedure performed. The discharge diagnosis was coded based on International Classification of Diseases, Ninth Revision, Clinical Modification (ICD-9-CM). We included acute myocardial infarction (AMI) (ICD-9-CM code 410.xx), coronary heart disease (CHD) (ICD-9-CM code 414.01) and ischaemic stroke (ICD-9-CM codes 433.xx and 434.xx). From among the NHI procedure codes, we included percutaneous transluminal coronary angioplasty (PTCA) and coronary artery bypass graft (CABG). The validity of the records in NHI claims by using these diagnosis and procedure codes has been studied and published, the results of which have shown high positive predictive values (PPV): 0.96-1.00 for ischaemic stroke, 0.88 for AMI, 0.83 for CHD, 0.92 for CABG and 0.98 for PTCA. More details were described in the Outcome Variables section. ${ }^{15} 16$

To comply with personal confidentiality regulations, patient identity data were encrypted and analysed anonymously. All results were reported in aggregated manner and no individual data shall be revealed.

\section{Baseline and covariate variables}

Patients' data 1 year prior to and during the hospitalisation were used as baseline variables for multivariate analysis. The baseline variables included age at admission, gender, discharge diagnosis, history of hyperlipidaemia before hospitalisation, Charlson Comorbidity Index, medication history, lipid testing before and during hospitalisation, accreditation level of healthcare organisation, index statin prescribing physicians' specialty and dosage of statin. In addition to the baseline variables, a time-varying covariate of whether lipid examination ever performed 1 month before statin prescription change was also included as an indicator to adjust for potential healthy adherer effect. ${ }^{17}$

\section{Treatment adherence and persistence variables}

We calculated separate measurements for statin adherence and persistence. All statin prescription records were retrieved from the index date, when the first statin was prescribed within 90 days of hospital discharge to one of the following: (1) the last record available of each patient, (2) subsequent ASCVD event that led to hospitalisation, or (3) end of database (on 31 December 2012), whichever occurred the earliest. The changing on adherence and persistence to statin therapy were captured along follow-up. We measured statin adherence using proportion of days covered (PDC) ${ }^{18}$ every 180 days. PDC was the number of days covered by statin prescription divided by the number of calendar days in each 180-day period (or number of days in the last period), which was a proportion ranged from 0 to 1 . A period with PDC $\geq 0.8$ was defined as good adherence, while PDC $<0.8$ as suboptimal adherence. The level at 0.8 or greater was selected as it was found to be the most consistently associated with clinical benefits in a previous review. ${ }^{19}$ Adherence was analysed as a binary variable and allowed for variation across multiple exclusive periods along follow-up of one patient.

Statin persistence was classified into four states based on the daily status of a patient with or without statin prescription covered along follow-up. ${ }^{20}$ All patients included in the cohort commenced as 'constant use' as statin initiated. A patient entered the 'non-persistence' state on the day when the patient failed to refill statin prescription that reached the 90th day after the end of the latest prescription. Within the 90-day window without statin prescription covered, the period was defined as 'recent stop'. If a statin prescription was refilled before the 90-day gap 
was reached, the patient returned to 'constant use' from 'recent stop'. During the 'non-persistence' state, if statin prescription resumed, the patient was moved into 'intermittent use' state and stayed for the rest of the follow-up. Figure 1 illustrates the diagram of persistence and adherence measurements.

\section{Outcome variables}

We ascertained two groups of outcomes, including subsequent rehospitalisation and in-hospital death due to $\mathrm{CV}$ events. For each group, three types of diagnosis were identified: (1) ASCVD composite event, (2) AMI diagnosis, and (3) ischaemic stroke diagnosis. The composite ASCVD event included one of the following: AMI (ICD-9-CM code 410.xx); ischaemic stroke (ICD-9-CM codes 433.xx and 434.xx); or stable CHD (ICD-9-CM code 414.01) with revascularisation (PTCA or CABG NHI procedure code) but without AMI diagnosis. According to the published validation studies of this coding, the PPV for ischaemic stroke was from 0.96 to 1.00 , for AMI 0.88, for revascularisation procedure of CABG 0.92 and for PTCA $0.98 .{ }^{15} 16$ The in-hospital death with AMI or stroke was validated as well with PPV of $0.79 .^{21}$

\section{Statistical analysis}

We used Cox proportional hazards model to analyse the association of statin prescription adherence and persistence on the study outcomes in respective model. Patients' data started at the index postdischarge statin prescription. Statin treatment adherence and persistence states were analysed as a time-dependent variable. We included baseline variables as time-fixed covariates and lipid test within 1 month prior to a statin prescription change as time-varying covariate to adjust for potential confounding on effect estimates. We presented adjusted HR (aHR) with associated $95 \%$ CI for each outcome. Two-sided p value $<0.05$ was considered statistically significant. We used Statistical Analysis System V.9.3 (SAS Institute) to carry out the analysis.

\section{RESULTS}

Figure 2 shows the patient selection flow for the study cohort. Within 90 days of hospital discharge for a new ASCVD, there were 185252 cases initiated statin treatment, and were followed for a total of 412752 patient-years, with a mean of 2.2 years, ranging from 1 to 7 years. Suboptimal adherence (PDC $<0.8$ ) accounted for $47 \%$ of the total follow-up duration. Distribution of follow-up duration by persistence states was: $50 \%$ in constant use, $13 \%$ in recent stop, $26 \%$ in non-persistence and $11 \%$ in intermittent use (table 2).

Baseline characteristics of patients and healthcare organisations were shown in table 1 and online supplementary file 1 . At index statin prescription, patients' mean age was 65.4 years, and $66 \%$ were male. During the 1 year prior to index hospitalisation, 44\% had hyperlipidaemia, $65 \%$ had received lipid testing and $47 \%$ had ever prescribed statins. The most frequent comorbidity was hypertension (68\%), followed by ischaemic heart disease (47\%) and diabetes (41\%). The mean Charlson comorbidity score was 1.79 . The top three concomitant medications were antiplatelet drugs (65\%), calcium channel blockers $(57 \%)$ and $\beta$ blockers (50\%). The most common ASCVD diagnosis at hospital discharge was ischaemic stroke (40\%), followed by CHD with revascularisation (37\%), and AMI with or without revascularisation (18\% and 5\%, respectively). During hospitalisation, $82 \%$ of the patients had received statin treatment and $88 \%$ underwent lipid testing. After discharge, most of the statins were initially prescribed in medical centres (51\%), followed by regional hospitals (31\%) and district hospitals (9\%). Most statins were prescribed by CV specialists (52\% CV medicine plus $4 \%$ CV surgery), followed by neurologists (29\%) (table 1).

In terms of outcome, a total of 50015 subsequent ASCVD rehospitalisations were found: among them 7986 were AMI and 15179 were ischaemic stroke. In-hospital death occurred in 2858 events, out of which 1273 and 1072 fatal events were due to AMI and ischaemic stroke, respectively (table 2). Table 3A summarises the results of association between statin adherence and risk of subsequent ASCVD. Good adherence to treatment (PDC $\geq 0.8$ ) was associated with statistically significant lower risk of subsequent hospitalisation. The aHRs $(95 \% \mathrm{CI})$ were 0.90 (0.87 to 0.92) for composite ASCVD outcome, 0.86 (0.81 to 0.92$)$ for AMI and 0.82 (0.78 to 0.86 ) for ischaemic stroke. Good adherence also associated with significantly lower risk of in-hospital death. The aHRs $(95 \% \mathrm{CI})$ were $0.59(0.53$ to 0.65$)$ for fatal ASCVD, 0.57 (0.49 to 0.66) for in-hospital death with AMI and 0.61 (0.52 to 0.73 ) for ischaemic stroke.

Table 3B summarises the results of statin persistence on subsequent ASCVD outcomes. Compared with constant use of statins, the other three lower persistence states (recent stop,

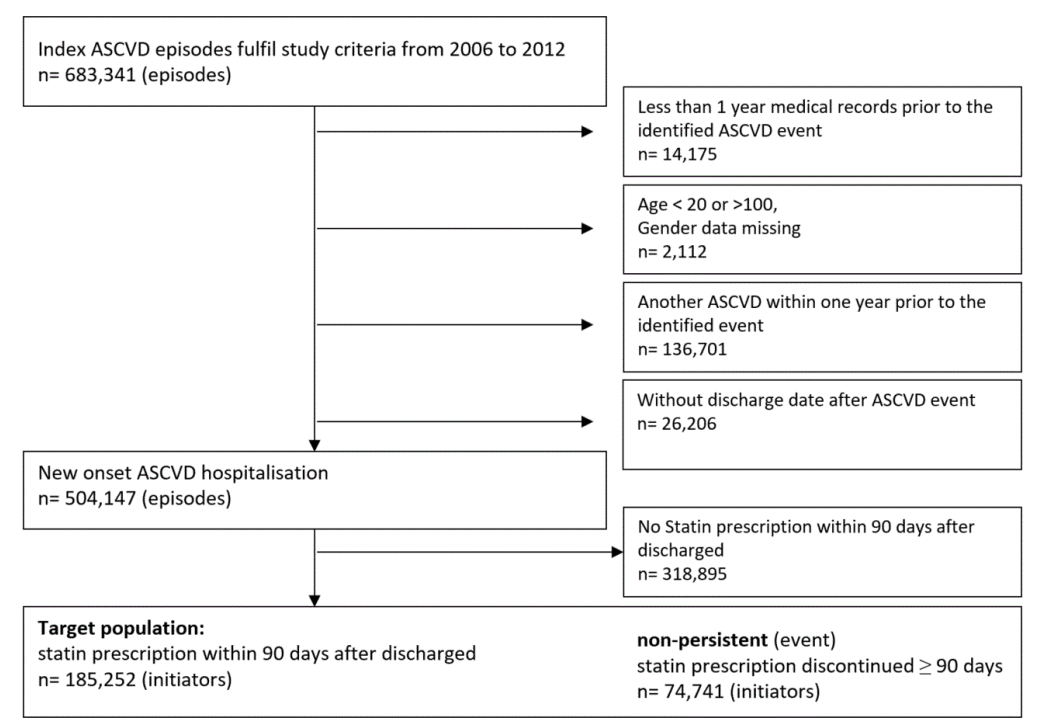

Figure 2 Selection diagram of study cohort. ASCVD, atherosclerotic cardiovascular disease. 
Table 1 Patient and treatment characteristics at baseline and initiation of statin treatment

\begin{tabular}{|c|c|c|}
\hline Variable & $\mathrm{n}^{*}$ & Proportion (\%) \\
\hline Total & 185252 & 100 \\
\hline Age $($ mean $\pm S D)$, years & $65.4 \pm 12$ & \\
\hline Male & 122881 & 66 \\
\hline \multicolumn{3}{|l|}{1 year prior to the ASCVD onset } \\
\hline Prior use of statins & 87191 & 47 \\
\hline Prior lipid examination & 119874 & 65 \\
\hline \multicolumn{3}{|l|}{ Comorbidity } \\
\hline $\begin{array}{l}\text { Charlson Comorbidity Index score } \\
\text { (mean } \pm \text { SD) }\end{array}$ & $1.79 \pm 1.8$ & \\
\hline Hyperlipidaemia & 81655 & 44 \\
\hline Hypertension & 126530 & 68 \\
\hline Ischaemic heart disease & 87031 & 47 \\
\hline Diabetes & 76390 & 41 \\
\hline COPD & 18112 & 10 \\
\hline \multicolumn{3}{|l|}{ Prior use of other medications } \\
\hline Antiplatelet & 119947 & 65 \\
\hline CCB & 104848 & 57 \\
\hline$\beta$ blockers & 92994 & 50 \\
\hline ARB & 72550 & 39 \\
\hline OAD & 68073 & 37 \\
\hline \multicolumn{3}{|l|}{ Baseline ASCVD hospitalisation } \\
\hline \multicolumn{3}{|l|}{ Type of ASCVD diagnosis } \\
\hline Ischaemic stroke & 74753 & 40 \\
\hline $\begin{array}{l}\text { Stable CHD receiving } \\
\text { revascularisation }\end{array}$ & 67779 & 37 \\
\hline AMI with PTCA/CABG & 33021 & 18 \\
\hline AMI without PTCA/CABG & 9699 & 5 \\
\hline In-hospital statin use & 151020 & 82 \\
\hline In-hospital lipid examination & 163329 & 88 \\
\hline \multicolumn{3}{|l|}{ Index date statin prescription } \\
\hline \multicolumn{3}{|l|}{ Healthcare institute accreditation level } \\
\hline Medical centre & 95326 & 51 \\
\hline Regional hospital & 58111 & 31 \\
\hline District hospital & 17129 & 9 \\
\hline Other hospital & 10440 & 6 \\
\hline Clinic & 4246 & 2 \\
\hline \multicolumn{3}{|l|}{ Physician specialty } \\
\hline Cardiovascular medicine & 96018 & 52 \\
\hline Cardiovascular surgery & 7540 & 4 \\
\hline Neurology & 53050 & 29 \\
\hline Metabolism and endocrinology & 7899 & 4 \\
\hline Internal medicine & 13798 & 7 \\
\hline Family medicine & 2971 & 2 \\
\hline Others & 3976 & 2 \\
\hline
\end{tabular}

*Except for the variables 'age' and 'Charlson Comorbidity Index score'. $A M I$, acute myocardial infarction; $A R B$, angiotensin receptor blocker; $A S C V D$ atherosclerotic cardiovascular disease; $C A B G$, coronary artery bypass graft; $C C B$, calcium channel blocker; $C H D$, coronary heart disease; $C O P D$, chronic obstructive pulmonary disorder; OAD, oral antidiabetic drug; PTCA, percutaneous transluminal coronary angioplasty.

non-persistence and intermittent use) were all associated with significantly higher risk of subsequent ASCVD rehospitalisation. The aHR for ASCVD composite outcome ranged from 1.13 to 1.26; for AMI from 1.21 to 1.60 ; and for ischaemic stroke from 1.26 to 1.47 (all $\mathrm{p}<0.05$ ). For in-hospital death, the patient in the state of recent stop with statin treatment (failed to refill prescription for less than 90 days) was associated with significantly higher fatal risk compared with that of patients in
Table 2 Study follow-up and outcomes

\begin{tabular}{lcc|}
\hline Follow-up & Patient-years & \\
\hline Total & 412752 & $100 \%$ \\
\hline By treatment persistence state & & \\
$\quad$ Constant use period & 208225 & $50 \%$ \\
\hline Recent stop period & 54051 & $13 \%$ \\
\hline Non-persistence period & 106961 & $26 \%$ \\
\hline Intermittent use period & 43516 & $11 \%$ \\
\hline By prescription adherence & & \\
\hline PDC $\geq 0.8$ (good adherence) & 218412 & $53 \%$ \\
\hline PDC $<0.8$ (suboptimal adherence) & 194340 & $47 \%$ \\
\hline Outcomes & Events (n) \\
\hline Rehospitalisation & & \\
\hline All ASCVD hospitalisation & 50015 \\
\hline AMI hospitalisation & 7986 \\
\hline Ischaemic stroke hospitalisation & 15179 \\
\hline In-hospital death & \\
\hline Any ASCVD in-hospital death & 2858 \\
\hline AMI in-hospital death & 1273 \\
\hline Ischaemic stroke in-hospital death & 1072 \\
\hline A
\end{tabular}

AMI, acute myocardial infarction; ASCVD, atherosclerotic cardiovascular disease; PDC, proportion of days covered.

the constant use state $(\mathrm{p}<0.05)$. The aHRs $(90 \% \mathrm{CI})$ were 1.42 (1.28 to 1.59$), 1.47$ (1.25 to 1.71$)$ and 1.54 (1.29 to 1.84 ) for fatal ASCVD, fatal AMI and fatal ischaemic stroke, respectively. The associations of non-persistence and intermittent use states with fatal events were not statically significant.

\section{DISCUSSION}

Statin therapy is important for secondary prevention of both non-fatal and fatal subsequent ASCVD events. The evidence has shown that the effect of statins persists if they are taken for long term. ${ }^{22}$ The use of high-intensity statins is recommended by several international treatment guidelines published in recent years. $^{1910}$ In this long-term follow-up of ASCVD discharge cohort, we found good adherence (PDC $\geq 0.8$ ) with statin treatment was associated with a $10 \%$ lower risk of subsequent ASCVD rehospitalisation. The strength of association was even stronger, specifically with AMI and ischaemic stroke (14\% and $18 \%$ lower risk, respectively); the strongest association was with in-hospital death (40\% lower risk). With respect to persistence, compared with the 'constant use' period, all the three lower persistence states (recent stop, non-persistence and intermittent use) were also associated with significantly higher risk of rehospitalisation outcomes across the composite ASCVD event as well as with AMI and ischaemic stroke events. These findings in the real-world practice for the effectiveness of use of statin for secondary prevention of ASCVD were consistent with the efficacy results of RCTs for statins compared with placebo on secondary prevention of $\mathrm{CV}$ events with the effect size of relative risk reduction in the range of $21 \%-30 \% .{ }^{9}$ Effectiveness of statin treatment on secondary prevention of ASCVD should be as expected if the medications were well taken.

In the analysis of statin persistence, we found that patients in 'recent stop' period were associated with significantly increased risk of both fatal and non-fatal events. Recent stop was defined as 'failed to refill statin within 90 days of end of last prescription,' this observation characterised the early emergence of recurrent ASCVD facing treatment discontinuation. It may relate to the 
Table 3A Effect of statin prescription adherence on time to subsequent ASCVD events

\begin{tabular}{|c|c|c|c|c|c|c|}
\hline \multirow{2}{*}{$\begin{array}{l}\text { Rehospitalisation } \\
\text { outcomes }\end{array}$} & \multicolumn{2}{|c|}{ All ASCVD } & \multicolumn{2}{|l|}{ AMI } & \multicolumn{2}{|c|}{ Ischaemic stroke } \\
\hline & $\mathrm{aHR}^{*}$ & $(95 \% \mathrm{Cl})$ & $\mathrm{aHR}^{*}$ & $(95 \% \mathrm{Cl})$ & $\mathrm{aHR}^{*}$ & $(95 \% \mathrm{Cl})$ \\
\hline \multicolumn{7}{|l|}{ Adherence } \\
\hline Suboptimal $($ PDC $<0.8)$ & Ref & & Ref & & Ref & \\
\hline Adherent (PDC $\geq 0.8)$ & 0.90 & $(0.87$ to 0.92$) \dagger$ & 0.86 & (0.81 to 0.92$) \dagger$ & 0.82 & $(0.78$ to 0.86$) \dagger$ \\
\hline \multirow{2}{*}{$\begin{array}{l}\text { In-hospital fatal } \\
\text { outcomes }\end{array}$} & \multicolumn{2}{|c|}{ Fatal ASCVD } & \multicolumn{2}{|c|}{ Fatal AMI } & \multicolumn{2}{|c|}{ Fatal ischaemic stroke } \\
\hline & $\mathrm{aHR}^{*}$ & $(95 \% \mathrm{Cl})$ & $a H R^{*}$ & $(95 \% \mathrm{Cl})$ & $\mathrm{aHR}^{*}$ & $(95 \% \mathrm{Cl})$ \\
\hline \multicolumn{7}{|l|}{ Adherence } \\
\hline Suboptimal $($ PDC $<0.8)$ & Ref & & Ref & & Ref & \\
\hline Adherent $(\mathrm{PDC} \geq 0.8)$ & 0.59 & $(0.53$ to 0.65$) \dagger$ & 0.57 & $(0.49$ to 0.66$) \dagger$ & 0.61 & $(0.52$ to 0.73$) \dagger$ \\
\hline
\end{tabular}

Table 3B Effect of statin prescription persistence on time to subsequent ASCVD events

\begin{tabular}{|c|c|c|c|c|c|c|}
\hline \multirow{2}{*}{$\begin{array}{l}\text { Rehospitalisation } \\
\text { outcomes }\end{array}$} & \multicolumn{2}{|c|}{ All ASCVD } & \multicolumn{2}{|l|}{ AMI } & \multicolumn{2}{|c|}{ Ischaemic stroke } \\
\hline & $\mathrm{aHR}^{*}$ & $(95 \% \mathrm{Cl})$ & $\mathrm{aHR}^{*}$ & $(95 \% \mathrm{Cl})$ & $\mathrm{aHR}^{*}$ & $(95 \% \mathrm{Cl})$ \\
\hline \multicolumn{7}{|l|}{ Persistence } \\
\hline Recent stop period & 1.16 & (1.13 to 1.19$) \dagger$ & 1.51 & $(1.42$ to 1.62$) \dagger$ & 1.38 & (1.32 to 1.45$) \dagger$ \\
\hline Non-persistence period & 1.13 & $(1.10$ to 1.16$) \dagger$ & 1.60 & $(1.49$ to 1.71$) \dagger$ & 1.47 & $(1.40$ to 1.54$) \dagger$ \\
\hline Intermittent use period & 1.26 & (1.21 to 1.31$) \dagger$ & 1.21 & $(1.09$ to 1.35$) \dagger$ & 1.26 & (1.17 to 1.35$) \dagger$ \\
\hline In-hospital fatal outcomes & $\mathrm{aHR}^{*}$ & $(95 \% \mathrm{CI})$ & $\mathrm{aHR}^{*}$ & $(95 \% \mathrm{Cl})$ & $\mathrm{aHR}^{*}$ & $(95 \% \mathrm{Cl})$ \\
\hline \multicolumn{7}{|l|}{ Persistence } \\
\hline Constant use period & Ref & & Ref & & Ref & \\
\hline Recent stop period & 1.42 & (1.28 to 1.59$) \dagger$ & 1.47 & $(1.25$ to 1.71$) \dagger$ & 1.54 & $(1.29$ to 1.84$) \dagger$ \\
\hline Non-persistence period & 0.91 & (0.82 to 1.01 ) & 0.86 & (0.73 to 1.01$)$ & 1.10 & (0.92 to 1.30$)$ \\
\hline
\end{tabular}

*Using Cox proportional hazards model and adjusted for age, sex, types of baseline ASCVD diagnosis, history of hyperlipidaemia 1 year before hospitalisation, Charlson Comorbidity Index 1 year before hospitalisation, medications 1 year before the enrolment date (antiplatelet, statin), number of lipid examination 1 year before and during hospitalisation, level of healthcare organisation, physician specialty of index statin, dosage of index statin and time-varying covariate of lipid examination 1 month before statin dose change.

$\dagger \mathrm{P}<0.05$.

aHR, adjusted HR; AMI, acute myocardial infarction; ASCVD, atherosclerotic cardiovascular disease; PDC, proportion of days covered.

theory of low-density lipoprotein (LDL) variability suggested by Bangalore $e t a l,{ }^{23}$ who found that patients with LDL fluctuation were subjected to higher risk of coronary and other CV events in a post hoc analysis of the Treat to New Targets trial. The discontinuation of statin treatment that defined the 'recent stop' period would likely be associated with LDL level rebounded in majority of patients. ${ }^{24}$ Our observation was in consistent with the review of 13 studies of statin non-adherence or discontinuation on $\mathrm{CV}$ outcomes, ${ }^{25}$ which found that patients with highly variable statin adherence were at particular risk for secondary ASCVD events. Moreover, within 1 month following the discontinuation of statin, the CV risk in diabetes and rheumatoid arthritis populations was significantly increased. ${ }^{25}$ This phenomenon may also be complementary with the findings of early reduction of $\mathrm{CV}$ risk within few weeks of statin initiation in RCTs of patients with post-AMI or those who underwent revascularisation procedures. 672627 To summarise, the result of 'recent stop' revealed the importance of the short-term acute effect of failure to maintain optimal treatment in patients with established ASCVD and emphasised the importance of sustained statin treatment. We noted that the 'non-persistence' and 'intermittent use' states had little effect on the fatal ASCVD outcomes, which suggested that once a patient survived the 'recent stop' period, the risk of fatal ASCVD outcomes diminished and became similar to that of the constant use state. It is worthy for further studies on this phenomenon in the future.
Several issues in the analysis of treatment effect in real world were considered in the design of this study. Reduction or changing of treatment compliance over time is common in real-world practice, especially for the long-term prevention of recurrent disease. In order to assess the treatment pattern more accurately, we used time-dependent Cox model to analyse adherence and persistence as time-varying variables. This method captured real-life drug use patterns allowed for variation in adherence and persistence over the 7 years of study follow-up. This is in contrast to other studies that used time-fixed measurement by pooling prescriptions across the whole follow-up period ${ }^{28}$ or taking a single time point observation ${ }^{29}$ that may subject to higher measurement error and result in biased estimate of effect. Furthermore, we analysed adherence and persistence in separate models to account for the two different constructs. ${ }^{20}$ PDC is commonly used to analyse treatment adherence and more consistently defined in previous studies; thus the result supported the existing published literature. ${ }^{13} 19{ }^{20}$ However, to calculate PDC, data need to be pooled across a period of time, the fluctuation within the calculation period will be smoothed out and the effect related to the change in prescription could not be captured. This drawback was recovered in our analysis of persistence and found recent stop associated with increased recurrent CV risk.

We used the new user design with all the subjects included in the analysis-initiated statin within the same 90 days' period of hospital discharge to minimise the potential selection by 
treatment indication. To control for potential confounders, we included extended variables covering factors of all the five dimensions related to medication adherence, described by the WHO. ${ }^{30}$ We included the age and sex for 'patient factors'; diagnosis of index ASCVD event, comorbidities and use of other medications for 'condition factors'; prior use of statin, history of lipid examination for 'therapy factors'; healthcare institute accreditation level and physician specialty for 'healthcare system factors'; and geographic location and year of index prescription for 'socioeconomic factors'. Most previous studies were not able to be as comprehensive with covering all the dimensions for adherence. ${ }^{13}$ In addition to the five dimensions of baseline factors, we included lipid testing prior to changing of statin prescription as another time-dependent variable to adjust for potential healthy adherer effect ${ }^{17}$ in the consideration of regular lipid testing, a potential indicator of better healthy-seeker behaviour.

Financial burden has been recognised as an important factor of statin adherence. ${ }^{31}$ One additional advantage of this study is that the claims data are taken from the NHI, which provides comprehensive coverage of medical services, including all the statins approved in Taiwan. This has minimised the potential impact of copayment affordability to adherence in this study. Also, the universal coverage of the NHI minimised the bias due to selection of patients or lost to follow-up due to change between health plans. The method used to define the study population and identify outcomes has been validated with high PPV in the NHI claims database. ${ }^{15} 16$

There were also limitations in this study. First, this study was based on the prescription records. We could not evaluate to what extent the prescribed medication was actually consumed by patients; thus, there could be potential exposure measure error, which may bias the estimates towards the null and result in conservative effect. Second, laboratory examination result of serum lipid level was not available in the database, which is an important parameter for the prescription of statins and predictor for CV outcomes. But this information might be important to the initiation of statin treatment; in the patients with established ASCVD and initiated treatment, their treatment should be maintained. Third, we could not capture lifestyle variables and socioeconomic factors, which may introduce confounding on the effect measurement. These could be factors that warrant further study to enhance treatment compliance and improve patient outcomes. Finally, we were not able to collect data on statin intolerance and side effects, although statins are generally well tolerated, the impact to the study result might be limited.

\section{CONCLUSIONS}

Good statin prescription adherence and persistence are associated with significant reduction in risk for ASCVD rehospitalisation in the context of secondary prevention. There is a need for further investigation into the factors associated with improving statin adherence and persistence and in developing effective strategies. As suggested by the WHO, medication adherence and persistence are related to multidimensional factors. To treat lipid disorders and reduce CV risk, we need to rely on more than the pharmaceutical effect. Most clinical guidelines recommend that the management of lipid disorders must incorporate lifestyle modifications and patient compliance behaviour. Our study suggests that optimising patient adherence and persistence behaviours is a core driver to improving patient outcomes.

Contributors JZL and SF have made substantial contributions to the conception and design of the study, interpretation of data and drafting of the article. WYS,
STH, CLL, STC and MSL have made substantial contributions to the conception and design of the study, acquisition of data, analysis and interpretation of data and drafting the article. VCT has made substantial contributions to the interpretation of data and drafting of the article. All authors were involved in revising the study critically for important intellectual content and gave final approval of the version to be submitted.

Funding This study was sponsored by Pfizer which is the manufacturer of Lipitor, the original brand of atorvastatin (Study ID A2581195). Editorial assistance was provided by APCER Life Sciences and funded by Pfizer.

Disclaimer Pfizer management was not involved in the review and interpretation of the results; the interpretation of the results was only from the authors. All analyses were done by the Formosan Medical Association; in fact, Pfizer did not have access to the data thus would not be able to, and did not, analyse the data. Because of this, the analyses were not repeated by another independent group. Pfizer follows the ICMJE standards, and this was clearly specified in the contract with the academic contractor Formosan Medical Association for this study: 'For all Publications, Investigator will comply with recognized ethical standards concerning publications and authorship, including Section II-'Ethical Considerations in the Conduct and Reporting of Research' of the Uniform Requirements for Manuscripts Submitted to Biomedical Journals, http://www.icmje.org/index.html\#authorship, established by the International Committee of Medical Journal Editors.'

Competing interests Among the authors of this study, WYS, JZL and SF are employees of Pfizer. STH, STC and MSL received research grant from Pfizer. VCT, who is an employee of APCER, received funding from Pfizer. CLL received consultancy fee from Pfizer.

Ethics approval The study protocol was reviewed by the Research Ethics Committee of the National Taiwan University, and was granted waiving of ethics approval and individual consent in accordance with local regulations.

Provenance and peer review Not commissioned; externally peer reviewed. Data sharing statement Data are available upon reasonable request.

\section{REFERENCES}

1. Stone NJ, Robinson JG, Lichtenstein AH, et al. 2013 ACC/AHA guideline on the treatment of blood cholesterol to reduce atherosclerotic cardiovascular risk in adults: a report of the American College of Cardiology/American heart association Task force on practice guidelines. J Am Coll Cardiol 2014;63:2889-934.

2. Mozaffarian D, Benjamin EJ, Go AS, et al. Heart disease and stroke Statistics-2016 update: a report from the American heart association. Circulation 2016;133:e38-60.

3. Dawber TR, Moore FE, Mann GV. Coronary heart disease in the Framingham study. Am J Public Health Nations Health 1957;47:4-24.

4. Kannel WB, McGee D, Gordon T. A general cardiovascular risk profile: the Framingham study. Am J Cardiol 1976;38:46-51.

5. Amarenco P, Bogousslavsky J, Callahan A, et al. High-Dose atorvastatin after stroke or transient ischemic attack. J Vasc Surg 2006;44:1374-59.

6. Cannon $\mathrm{CP}$, Braunwald $\mathrm{E}, \mathrm{McC}$ abe $\mathrm{CH}$, et al. Intensive versus moderate lipid lowering with statins after acute coronary syndromes. N Engl J Med 2004;350:1495-504.

7. Schwartz GG, Olsson AG, Ezekowitz MD, et al. Effects of atorvastatin on early recurrent ischemic events in acute coronary syndromes: the MIRACL study: a randomized controlled trial. JAMA 2001;285:1711-8.

8. Baigent C, Keech A, Kearney PM, et al. Efficacy and safety of cholesterol-lowering treatment: prospective meta-analysis of data from 90,056 participants in 14 randomised trials of statins. Lancet 2005;366:1267-78.

9. NICE clinical guideline CG181. Lipid modification: cardiovascular risk assessment and the modification of blood lipids for the primary and secondary prevention of cardiovascular disease. clinical guideline. methods, evidence and recommendations. NICE clinical guideline CG181 lipid modification: cardiovascular risk assessment and the modification of blood lipids for the primary and secondary prevention of cardiovascular disease clinical guideline methods, evidence and recommendations. London; 2014.

10. Catapano AL, Graham I, De Backer G, et al. 2016 ESC/EAS Guidelines for the Management of Dyslipidaemias: The Task Force for the Management of Dyslipidaemias of the European Society of Cardiology (ESC) and European Atherosclerosis Society (EAS) Developed with the special contribution of the European Assocciation for Cardiovascular Prevention \& Rehabilitation (EACPR). Atherosclerosis 2016;253:281-344.

11. Hippisley-Cox J, Coupland C. Effect of statins on the mortality of patients with ischaemic heart disease: population based cohort study with nested case-control analysis. Heart 2006;92:752-8.

12. Kumbhani DJ, Steg PG, Cannon CP, et al. Adherence to secondary prevention medications and four-year outcomes in outpatients with atherosclerosis. Am J Med 2013;126:693-700. 
13. De Vera MA, Bhole V, Burns LC, et al. Impact of statin adherence on cardiovascular disease and mortality outcomes: a systematic review. Br J Clin Pharmacol 2014;78:684-98.

14. National Health Insurance Administration, Ministry of Health and Welfare, Taiwan, R.O.C. National health insurance annual report 2014-2015; 2014.

15. Cheng C-L, Kao Y-HY, Lin S-J, et al. Validation of the National health insurance research database with ischemic stroke cases in Taiwan. Pharmacoepidemiol Drug Saf 2011;20:236-42.

16. Cheng $C-L$, Lee $C-H$, Chen $P$-S, et al. Validation of acute myocardial infarction cases in the National health insurance research database in Taiwan. J Epidemiol 2014;24:500-7.

17. Shrank WH, Patrick AR, Brookhart MA. Healthy user and related biases in observational studies of preventive interventions: a primer for physicians. J Gen Intern Med 2011;26:546-50.

18. Choudhry NK, Shrank WH, Levin RL, et al. Measuring concurrent adherence to multiple related medications. Am J Manag Care 2009;15:457-64.

19. Simpson RJ, Mendys P. The effects of adherence and persistence on clinical outcomes in patients treated with statins: a systematic review. J Clin Lipidol 2010;4:462-71.

20. Cramer JA, Roy A, Burrell A, et al. Medication compliance and persistence: terminology and definitions. Value Health 2008;11:44-7.

21. Cheng C-L, Chien H-C, Lee C-H, et al. Validity of in-hospital mortality data among patients with acute myocardial infarction or stroke in national health insurance research database in Taiwan. Int J Cardiol 2015;201:96-101.

22. Collins R, Reith C, Emberson J, et al. Interpretation of the evidence for the efficacy and safety of statin therapy. Lancet 2016;388:2532-61.
23. Bangalore S, Fayyad R, Laskey R, et al. Lipid lowering in patients with treatmentresistant hypertension: an analysis from the treating to new targets (TnT) trial. Eur Heart J 2014;35:1801-8.

24. Mann DM, Glazer NL, Winter M, et al. A pilot study identifying statin nonadherence with visit-to-visit variability of low-density lipoprotein cholesterol. Am J Cardiol 2013:111:1437-42.

25. Phan K, Gomez Y-H, Elbaz L, et al. Statin treatment non-adherence and discontinuation: clinical implications and potential solutions. Curr Pharm Des 2014;20:6314-24.

26. Sever PS, Dahlöf B, Poulter NR, et al. Prevention of coronary and stroke events with atorvastatin in hypertensive patients who have average or lower-than-average cholesterol concentrations, in the Anglo-Scandinavian Cardiac Outcomes Trial-Lipid Lowering Arm (ASCOT-LLA): a multicentre randomised controlled trial. Lancet 2003;361:1149-58.

27. Patti G, Pasceri V, Colonna G, et al. Atorvastatin pretreatment improves outcomes in patients with acute coronary syndromes undergoing early percutaneous coronary intervention: results of the ARMYDA-ACS randomized trial. J Am Coll Cardiol 2007;49:1272-8

28. Li Y-C, Huang W-L. Effects of adherence to statin therapy on health care outcomes and utilizations in Taiwan: a population-based study. Biomed Res Int 2015;2015:149573.

29. Ho PM, Spertus JA, Masoudi FA, et al. Impact of medication therapy discontinuation on mortality after myocardial infarction. Arch Intern Med 2006;166:1842-7.

30. WHO. Adherence to long-term therapies. Evidence for action, 2003. Available: http:// apps.who.int/iris/bitstream/10665/42682/1/9241545992.pdf

31. Ellis JJ, Erickson SR, Stevenson JG, et al. Suboptimal statin adherence and discontinuation in primary and secondary prevention populations. J Gen Intern Med 2004; 19:638-45. 